\title{
Acute salivary gland inflammation associated with systemic lupus erythematosus
}

\author{
W. A. KATZ AND G. E. EHRLICH \\ The Arthritis Center, Albert Einstein Medical Center-Moss Rehabilitation Hospital; Temple University School \\ of Medicine, Philadelphia, Pennsylvania, U.S.A.
}

The parotid gland may enlarge during the course of systemic lupus erythematosus (SLE). Shearn and Pirofsky (1952) were the first to draw attention to this association while studying a group of 34 patients who had systemic lupus erythematosus. They discovered sialoadenitis characterized by chronic, nonsuppurative inflammation unilaterally in one and bilaterally in two patients. Morgan (1954) reported systemic lupus erythematosus in a patient with the Sjögren-Mikulicz syndrome, and Harvey, Shulman, Tumulty, Conley, and Schoenrich (1954) described a 13-year-old Negro girl who developed chronic parotid swelling and eventually died from classical lupus erythematosus.

Heaton (1959) claimed that most of his 28 cases of Sjögren's syndrome had other findings suggestive of SLE including ten with positive LE preparations. In his text, Dubois (1966) noted six of 520 cases with SLE (1.1 per cent.) as having extreme enlargement of the parotid, submaxillary, and/or lacrimal glands. Although none of these cases was acute, one, a 19-year-old Negro girl, was admitted because of parotid swelling of only 4 weeks' duration associated with a flare of her generalized disease. All other authors (Futcher, 1959; Bain, 1960) have likewise reported subacute or chronic salivary gland enlargement not parallelling systemic disease activity. As a matter of fact, Heaton (1959) concluded that Sjögren's syndrome is a chronic, relatively benign form of systemic lupus erythematosus. We report three cases in which acute inflammation of the salivary glands reflected lupus activity.

\section{Case reports}

Case 1, a 15-year-old Black girl, was first admitted to the Albert Einstein Medical Center on February 19, 1969, with complaints of multiple joint pain, morning stiffness, generalized weakness, and fatigue for 2 years. She had developed fever accompanied by pain and swelling at the angle of the jaws, and a diagnosis of 'mumps' was made by her physician, even though there had been no known exposure to infection. The symptoms disappeared within a few days; however, the patient subsequently became aware of exertional dyspnoea and pruritic papular eruptions on the flexor surfaces of the extremities and face.

Physical examination showed multiple pigmented macular areas covering the arms, legs, and malar region. There was clinical evidence of pericardial and pleural effusions, and tenderness was present in the right and left costovertebral angles. The liver, spleen, and kidneys were not palpable. The salivary glands were not enlarged or tender. There was a synovitis of both wrists.

Chest $x$ rays confirmed the existance of a small left pleural effusion with moderate globular cardiomegaly. During the ensuing 3 weeks when she was receiving full dose salicylates and rest in bed the joint pain gradually disappeared. She became less dyspnoeic, and the heart became progressively smaller.

After her discharge she remained well until 1 week before her second admission to hospital on April 9, 1969, when she developed a marked swelling in the submandibular region, which progressively enlarged to the angle of the jaw. This was associated with anorexia and progressive generalized weakness.

\section{Examination}

She was acutely ill, complaining of severe pain in the jaw. Temperature was $105^{\circ} \mathrm{F}$. There was a circumscribed swelling of the entire left side of the face and the left submandibular area, which was hot, red, and tender (Fig. 1, opposite). The submandibular nodes and anterior cervical nodes on the left were enlarged and tender. The heart again was found to be enlarged, and a pleural effusion was noted at the left base. No evidence of arthritis was present.

Mandibular $x$ rays showed considerable soft tissue swelling of the mandible and neck, but no calculi or osseous abnormalities were discovered. A sialogram revealed tertiary and quaternary intraductal ectasia, a picture most commonly seen in Sjögren's syndrome.

\section{Laboratory investigations}

Repeated culturing of the salivary gland ducts and blood produced no micro-organisms. Haemoglobin was $7 \cdot 5 \mathrm{~g}$. per cent.; haematocrit, 24; white cell count, 7,600 with 


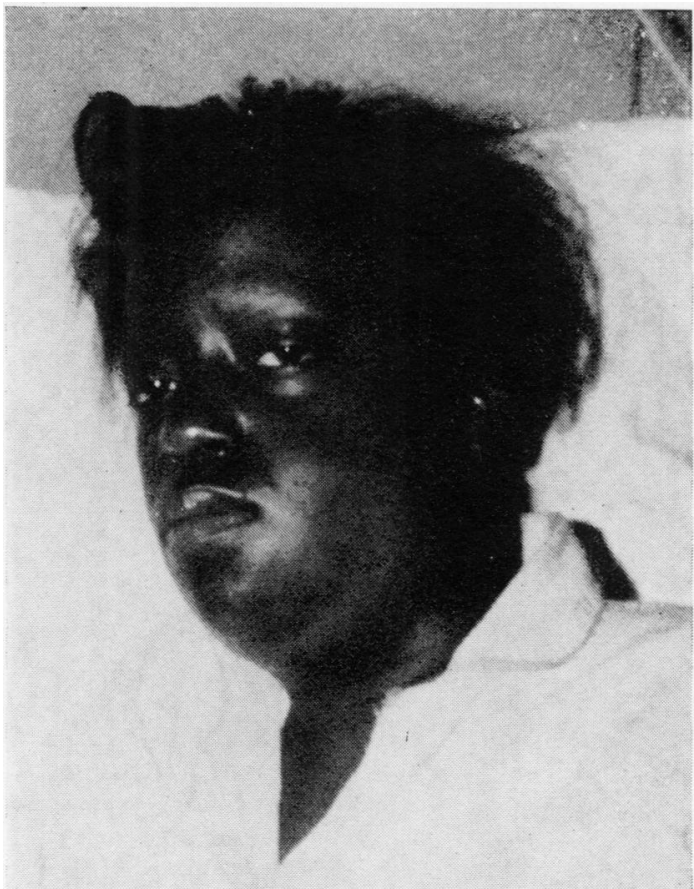

FIG. 1 15-year-old girl with acute inflammation of the submandibular gland

48 per cent. segmented neutrophils, 45 per cent. lymphocytes, and 7 per cent. monocytes. Wintrobe erythrocyte sedimentation rate $55 \mathrm{~mm}$./1st hour.

LE preparations positive. Antinuclear antibodies demonstrable at a 10:1 dilution using an immunofluorescent technique with mouse liver as nuclear substrate. Anti-DNA antibody, determined by the method of Pincus, Schur, Rose, Decker, and Talal (1969), significantly positive at 75 per cent. Serum complement $21 \cdot 2 \mathrm{CH}_{50}$ units/ml. (normal 20-45); $\mathrm{C}_{3}$ complement $95 \mathrm{mg}$. per cent. (normal 100-190). Latex-fixation test for rheumatoid factor negative. Urine analysis normal. Inulin clearance reduced to $78 \mathrm{ml} . / \mathrm{min}$. Reticulocyte count, platelet count, sickle cell preparation, blood sugar, BUN, creatinine, uric acid, bilirubin, prothrombin time, SGOT, SGPT, LDH, alkaline phosphatase, glucose-6-phosphate dehydrogenase, VDRL, and bone marrow aspirate were all unremarkable.

\section{Diagnosis}

An initial diagnosis of acute systemic lupus erythematosus with lupus sialoadenitis and secondary bacterial infection was made.

\section{Therapy}

A regimen of full-dose salicylates and ampicillin followed by nafcillin was begun.

\section{Progress}

However, despite this therapy, she continued to have fevers as high as $104^{\circ} \mathrm{F}$. for 3 days. Stenson's duct was oedematous and an attempt to cannulate it was unsuccessful. Small amounts of bloody fluid aspirated from the submandibular mass were sterile on culture. On the 4th day in hospital, methylprednisolone was started in daily doses of $64 \mathrm{mg}$.; defervescence of her symptoms and fever resulted within 8 hours. From that time on, she remained afebrile, and within 18 days the parotid gland shrank to normal size.

Biopsy of the submaxillary glands on the 14th day in hospital disclosed atrophy of the salivary glands and distended ducts. There was a background of stromal infiltration of mononuclear inflammatory cells. Some of the ducts contained loose inspissated material. The appearance was thought to be consistent with ranula and chronic sialoadenitis involving minor salivary glands (Figs 2 and 3, opposite).

Biopsy of the submandibular gland on the 22nd day in hospital yielded necrotic glandular tissue with intense acute and chronic inflammation.

\section{Termination}

$2 \frac{1}{2}$ years later, the patient died from a severe lobar pneumonia. The salivary glands were not clinically involved at the time of her death. The submaxillary glands at autopsy showed a number of chronic inflammatory cells, mostly lymphocytes, around some of the ducts. Otherwise, the gland was unremarkable. The pancreas showed no abnormality.

Case 2, a 54-year-old white woman, developed pain, swelling, and redness of the right wrist, proximal interphalangeal joints of the left hand, and left knee some years before her admission to hospital. The joint pain recurred intermittently and was often associated with episodes of alopecia and transient ulcerations of the soft and hard palate. 8 months before admission, an erythematous malar eruption was discovered. Chloroquine, $250 \mathrm{mg}$. twice daily, and prednisone, $30 \mathrm{mg}$. daily, caused a prompt amelioration of her symptoms. During the 2 months preceding admission, the dose of prednisone was gradually reduced to $15 \mathrm{mg}$. a day. 2 days after the dose was further lowered to $12.5 \mathrm{mg}$. daily, the patient again noted joint pain and swelling. This time it was associated with acute pain and swelling at both angles of the jaw. There was no known exposure to mumps.

\section{Examination}

The patient did not appear acutely ill. The parotid glands were found to be enlarged, hardened, and swollen. The overlying skin was reddened. Marked tenderness was elicited bilaterally. There was obvious difficulty in opening the mouth. She was afebrile. There was slight pain on motion and soft tissue swelling of the left wrist. The remainder of the examination was within normal limits.

\section{Laboratory investigations}

Haemaglobin 12.5 g.; haematocrit 36 per cent.; white cell count, 4,500 with 60 per cent. segmented neutrophils, 35 per cent. lymphocytes, and 5 per cent. monocytes. The LE preparation was positive and antinuclear antibodies at a 10:1 dilution were demonstrable using an immunofluorescent technique with mouse liver as nuclear substrate. The erythrocyte sedimentation rate was $45 \mathrm{~mm}$./ 1st hour (Westergren).

\section{Treatment}

The dose of prednisone was increased to $25 \mathrm{mg}$. a day.

Result

Within 24 hours, the patient was totally asymptomatic, and within 48 hours the parotid swelling completely disappeared. 


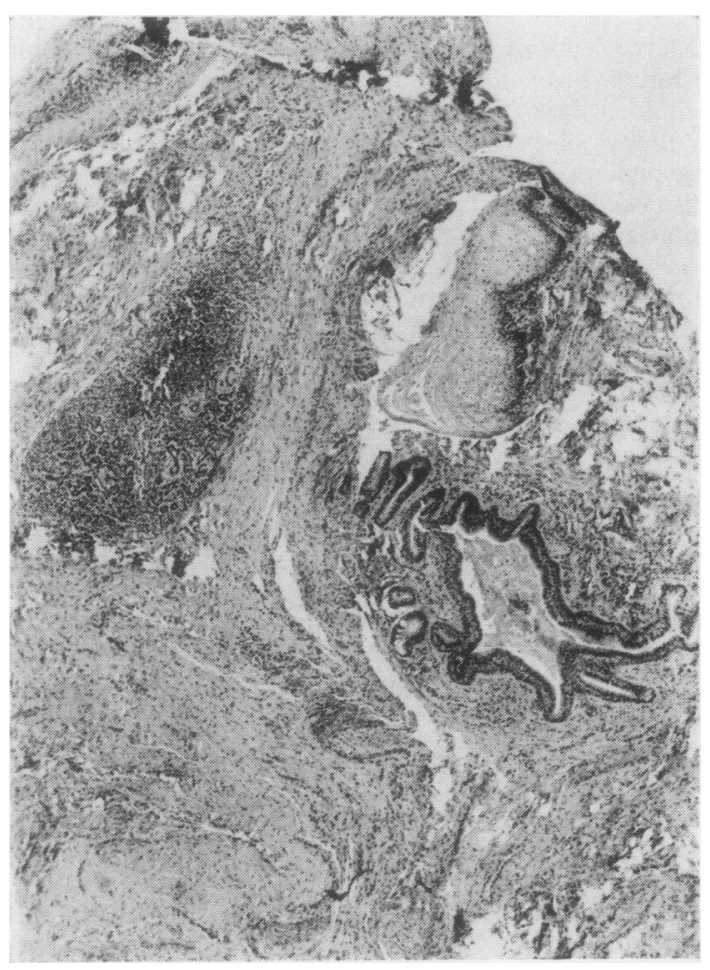

FIG. 2 Photomicrograph, showing chronic sialoadenitis of mandibular gland. A large distended duct contains inspissated material. Haematoxylin and eosin. Original magnification $\times 30$

Case 3, a 25-year-old Black woman, was admitted to the Albert Einstein Medical Center on December 1, 1968, with a 4-month history of slowly progressive polyarthralgia involving primarily the elbows, wrists, and knees, associated with morning stiffness lasting 3 to 4 hours. In addition, she noted increasing fatigue and generalized weakness. Only slight relief was obtained from aspirin. She could recall no facial rash, mouth ulcerations, alopecia, or pleuritic chest pain. There was no known exposure to mumps.

\section{Examination}

Temperature $101 \cdot 8^{\circ} \mathrm{F}$. There was noticeable periorbital oedema and moderate swelling and tenderness over both parotid glands. The thyroid gland was found to be diffusely enlarged. There was synovitis of the second and third metacarpophalangeal joints and all the interphalangeal joints of the right hand. Other observations were within normal limits.

\section{Laboratory investigations}

Urine analysis showed numerous red blood cells and a few white cells but no casts. Haemaglobin $9.1 \mathrm{~g}$. per cent.; haematocrit, 29 per cent.; white cell count, 2,350 , with 13 per cent. polymorphonuclears, 77 per cent. lymphocytes, 4 per cent. eosinophils, 2 per cent. monocytes, and two atypical lymphocytes. The erythrocyte sedimentation rate was $100 \mathrm{~mm} / 1 \mathrm{st} \mathrm{hr}$ (Westergren). Three LE preparations were positive (LE preparations done 3 weeks pre-

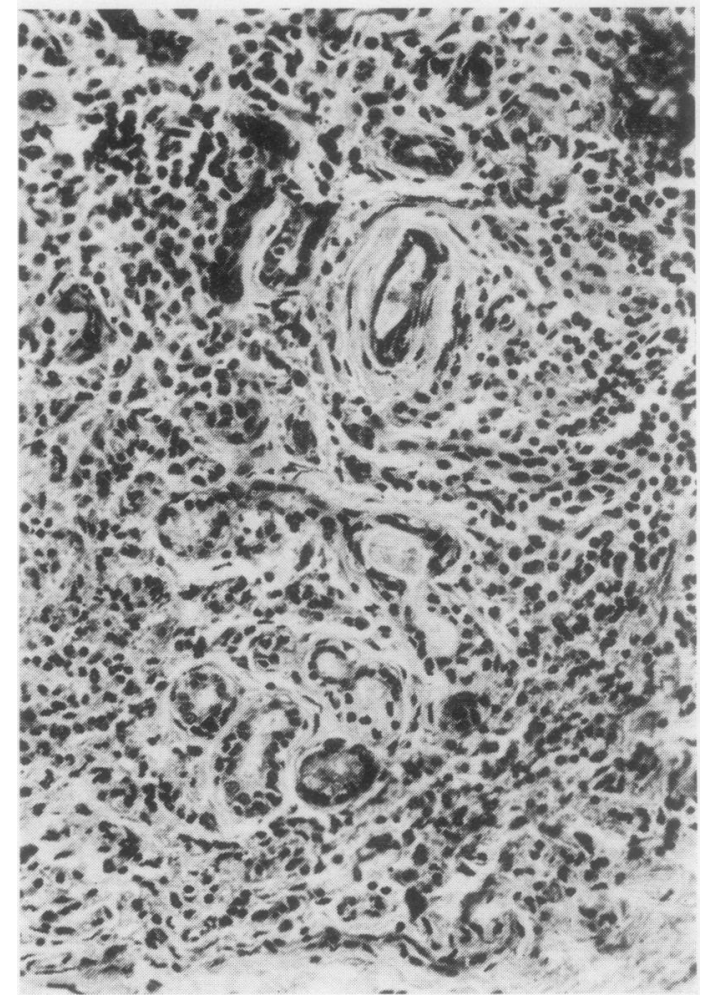

FIG. 3 Salivary gland duct walls are oedematous. Acinar tissue is replaced by mononuclear cells admixed with occasional plasma cells $\times 225$

viously had been negative). Platelet count 204,000; reticulocyte count 1.2 per cent. Antinuclear antibodies at a 10:1 dilution were demonstrable using an immunofluorescent technique with mouse liver as nuclear substrate. Anti-DNA borderline at 11.5 per cent. Serum complement $21.2 \mathrm{CH}_{50}$ u per ml., $\mathrm{C}_{3}$ complement component $74 \mathrm{mg}$. per cent. (normal 100-190). Serum electrophoresis disclosed diminished albumin and an increased broad-based polyclonal gamma globulin. Serum uric acid $8.6 \mathrm{mg}$. per cent. Glucose, BUN, creatinine, bilirubin, prothrombin time, SGPT, LDH, serum iron were all within normal limits. Latex-fixation test negative; thyroid globulin antibody tests weakly positive. Coombs's test negative. Bone marrow examination normal. Renal function studies showed inulin clearance diminished to $27 \mathrm{ml}$./min. and PAH clearance diminished to $415 \mathrm{ml}$./min. This was felt to be consistent with an active glomerulopathy. A renal biopsy disclosed local cellularity and degeneration consistent with early lupus erythematosus.

\section{Treatment and Result}

Methylprednisolone, $40 \mathrm{mg}$. per day, resulted in marked amelioration of all symptoms after 3 days. The parotid glands reverted to normal size and were non-tender.

\section{Discussion}

Systemic lupus erythematosus is usually classified as a specific disease, while Sjögren's syndrome has been 
accepted as a conjunction of symptoms, signs, and laboratory abnormalities accompanying several connective tissue disorders. Enlargement of the salivary glands has been well recognized in systemic lupus erythematosus but, except one case reported by Dubois (1966), all were chronic without clinical signs of inflammation and did not noticeably wax and wane with fluctuation of lupus activity. These enlargements have been thought to produce Sjögren's syndrome, pathologically characterized by lymphocytic infiltration replacing acinar tissue, reduction in acini, and the presence of myoepithelial islands with eventual fibrosis and glandular ectasia. Although these chronic manifestations of Sjögren's syndrome are well known, there is little understanding of its development. The three cases we have described are, therefore, of interest as possibly representing such an acute stage of Sjögren's syndrome.

Another explanation is that, in our cases, the salivary gland inflammation represents actual lupus involvement. The rapid onset of inflammation of the salivary glands occurring concomitantly with lupus activity in other organs and the dramatic response to corticosteroids in all cases lends credence to this hypothesis. Because the salivary glands are not routinely biopsied, there are no pathological studies of the acutely inflamed salivary gland in systemic lupus erythematosus; but autopsy studies suggest that the pancreas, 'sister organ' to the parotid gland, is often abnormal (Harvey and others, 1954). Most of the time, an arteritis is found, leading to chronic changes including atrophy and ectasia.

In our cases, the salivary glands enlarged concomitantly with exacerbations of systemic lupus erythematosus and reverted to normal size during drug-induced remissions of the disease. In the first case, the submaxillary gland was the site of predominant involvement; in the other two, the parotids. Clinically active inflammation was characterized by pain, swelling, tenderness, and warmth (and even redness in the first two cases) (Fig. 1). Although no attempt was made to rule out infection or obstruction in the second and third cases, the bilateral involvement and rapid response to corticosteroids alone in the absence of antibiotic therapy makes the possibility of bacterial sialoadenitis remote. None of the patients was exposed to mumps. Though virus studies were not performed to rule out epidemic parotitis, this diagnosis was not considered likely on clinical grounds. Negative cultures of the blood, glandular aspirates, and ductal secretions in the first case helped to eliminate an infective basis. Treatment with antibiotics failed to affect the sialoadenitis favourably, providing further evidence against infectious aetiology. Cannulation of the duct and sialogram failed to yield any evidence of obstruction. Certain drugs, such as potassium iodide, phenylbutazone, guanethedine, and methimizole, have been known to induce parotid swelling, but none of the patients was receiving any of these medicaments. In one patient, the parotid gland enlarged during a period of corticosteroid reduction. Biopsy of the submaxillary gland in the first case showed only non-specific inflammatory changes with no suppuration. It seems likely that the acute sialoadenitis may be a manifestation of acute systemic lupus erythematosus, possibly representing the acute stage of Sjögren's syndrome.

\section{Summary}

Three patients are presented in whom acute sialoadenitis accompanied flaring or active systemic lupus erythematosus. It is possible that these may represent the early stages of what would later be diagnosed as Sjögren's syndrome, the relationship of which with systemic lupus erythematosus has been well established.

\section{References}

BaIN, G. O. (1960) Canad. med. Ass. J., 82, 143 (The pathology of Mikulicz-Sjögren disease in relation to disseminated lupus erythematosus)

DuвoIs, E. (1966) 'Lupus Erythematosus' McGraw-Hill Book Company, New York

Futcher, P. H. (1959) Bull.Johns Hopk. Hosp., 105, 97 (Enlargement and round cell infiltration of the salivary glands associated with systemic disease)

Harvey, A. M., Shulman, L. E., Tumulty, P. A., Conley, C. L., and Schoenrich, E. H. (1954) Medicine (Baltimore), 33, 291 (Systemic lupus erythematosus: Review of the literature and clinical analysis of 138 cases)

Heaton, J. M. (1959) Brit. med.J., 1, 466 (Sjögren's syndrome and systemic lupus erythematosus)

Morgan, W. S. (1954) New Engl. J. Med., 251, 5 (The probable systemic nature of Mikulicz's disease and its relation to Sjögren's syndrome)

Pincus, T., Schur, P. H., Rose, J. A., Decker, J. L., and Talal, N. (1969) Ibid., 281, 701 (Measurement of serum DNA-binding activity in systemic lupus erythematosus)

Shearn, M. A., AND Pirofsky, B. (1952) Arch intern. med., 90, 790 (Disseminated lupus erythematosus-Analysis of 34 cases) 
of contents and an index of diseases, but the international bibliography is kept short. In view of the rising cost of everything we buy, the price of $£ 3.85$, in soft covers, is not excessive.

DAVID PREISKEL

A Practical Approach to Arm Pain. Edited by Meredith S. Hale. 1971. Pp. 103, 41 figs. Thomas, Springfield, Ill. (\$8.25)

The title and indeed the introduction to this book encourage the reader to expect a practical approach to the identification of pain felt in the arm. In the sense that five contributors and the editor between them outline the main causes of arm pain, the reader will not be disappointed. However, there is little sign of editorial effort to relate the many causes listed to clinical reality. Thus the shoulder (gleno-humeral) joint is dismissed with the brief statements that it is uniquely free from osteoarthritis and degenerative conditions and that a frozen shoulder may be found with cervical radiculopathy, while peripheral neuropathy as a cause of arm pain commands several pages including a classification of some 55 causes. Even myasthenia gravis is mentioned as a cause of arm pain!

The editor's interest in posturally-induced pain is apparent from the inclusion of a whole chapter on scapular traction and related syndromes and of one on their treatment by a combination of shoulder shrugging exercises, cervical posturing, and neck flexor exercises. The treatment of the common causes of arm pain receives scant mention.

None the less, the different approaches of an orthopaedic surgeon, a neurologist, a neurosurgeon, a vascular surgeon, and an internist to various aspects of arm pain are well illustrated by the first five chapters of this book, and these in combination with the editor's chapters on median, ulnar, and radial pressure neuropathies, including consideration of relevant electromyographic techniques, are worth study by rheumatologists to whom arm pain is a common problem and sometimes a diagnostic trap.

A. T. RICHARDSON

\title{
Notes
}

\section{International Congress of Rheumatology}

\author{
Kyoto, Japan, September 30 to October 6, 1973
}

The XIII International Congress of Rheumatology, sponsored by the Ligue Internationale contre le Rhumatisme and organized by the Japan Rheumatism Association and Foundation, will be held at Kyoto, Japan, on September 30 to October 6, 1973, under the presidency of Prof. Y. Oshima. Meetings of the PanAmerican, European, and South-east Asia and Pacific Leagues will be held on September 30. The second International Geigy Rheumatism Prize will be awarded at the final session on October 6. An extensive programme of lectures, discussions, and symposia has been arranged.

Further information may be obtained from the Secretariat, Japan Convention Services Inc., 3-23 7-chome, Roppongi, Minato-ku, Tokyo, 106, Japan.

Particulars of travel arrangements from Europe, including various tours of the Far East, may be obtained from H. Stulz, Boîte postale 149, CH 4010, Basel, Switzerland.

\section{Pan-American Congress on Rheumatic Diseases}

Toronto, Canada, June 16-21, 1974

A preliminary notice of this congress, to be held under the presidency of Prof. M. A. Ogryzlo, announces that the last date for submission of abstracts for inclusion in the scientific programme is January 15, 1974. Communications should be addressed to: The Congress Secretariat, 45, Charles Street East, Toronto 285, Ontario, Canada.

\section{Corrigendum}

In the article entitled 'Acute Salivary Gland Inflammation associated with Systemic Lupus Erythematosus' by W. A. Katz and G. E. Ehrlich Annals (1972), 31, 384;

On page 387, col. 1, 1. 9, for 'thought to produce' please read 'thought to be produced by Sjögren's syndrome'. 